ions. The catabolic response to surgery leads to further loss. When potassium has to be given by intravenous injection, it should not be of greater concentration than 40 m.equiv./l.

It is generally agreed that fæcal excretion of iron represents unabsorbed iron. Impaired absorption has been observed in the steatorrhœea syndrome even in patients with neither achlorhydria nor overt diarrhœa. Moderate impairment can often be countered by oral administration of iron salts, but severe cases require intravenous injections.

The pathogenesis of osteomalacia in association with steatorrhoea is complicated. First, the presence of excessive fatty acid in the bowel leads to the formation of calcium soaps the calcium of which is not available for absorption; this can be improved by a low fat diet. Also, vitamin D is poorly absorbed, and further improvement of absorption can be induced by parenteral vitamin $\mathrm{D}$ or very large doses by mouth. The prolonged treatment of peptic ulceration with large amounts of milk and alkalis can lead to calcium intoxication and, in severe cases, renal failure associated with calcification of the renal tubules.

Prof. R. B. Hunter (Department of Pharmacology and Therapeutics, Queen's College, Dundee) reviewed the method of elinical presentation of fifteen cases of idiopathic steatorrhœa recently studied in the Therapeutics Unit, Maryfield Hospital, Dundee. In less than half these cases was diarrhoa the predominant symptom though all complained of tiredness and loss of appetite. The patients learned to control their own diarrhoea by reducing their fat intake. Cases of apparent iron-deficiency anæmia with malabsorption of fat, macrocytic anæmia or megaloblastic anæmias responding to folic acid were shown. Two cases of extreme calcium deficiency were considered in detail, and the problems of finding an effective means of calcium replacement in the face of impaired alimentary absorption were considered.

Food allergy was discussed by Dr. J. A. Milne (Department of Pathology, University of Glasgow), who said that, in order to discuss food allergy, it is necessary to consider first certain basic facts concerning allergic reactions and the immunological basis of allergy. When an antigen first enters the body, it stimulates the formation of specific antibodies. Some of these antibodies tend to persist in their free state in the circulating blood (circulating antibodies), and this would appear to constitute immunity. Others become incorporated into the substance of cells and become 'sessile' antibodies or fixed antibodies, and so the allergic state is established. Why some people develop sessile antibodies and thus become hypersensitive is completely unknown. Various sites may be affected-for example, the nasal mucosa and conjunctiva, the skin, etc.and tissues so affected are known as 'shock organs'. What actually takes place when a tissue containing sessile antibodies comes in contact with its specific antigen is not known. There is considerable experimental and clinical evidence to suggest that histamine or a closely related substance is released.

Proof of the existence of food allergy depends on the demonstration of a cause-and-effect relationship between the ingestion of a given food and the production or accentuation of the patient's symptoms. The use of skin tests, either by the scratch or intradermal technique using concentrated extracts of foods, may occasionally be of help, although on the whole the results tend to be disappointing. For- tunately, many persons with food allergy, as well as having sessile antibodies, have some circulating antibodies. This allows the use of the PrausnitzKustner reaction for diagnostic purposes.

The ideal treatment of food allergy is, of course, the avoidance of the offending food, if this be known. The most widely used drugs at present are the antihistamines, and the results with them other than in papular urticaria in children are rather disappointing. Cortisone and adrenocorticotrophic hormone have been used with good results, although the symptoms tend to recur with increased severity once the drug is withdrawn.

\section{FERROELECTRIC PROPERTIES OF SINGLE CRYSTALS}

A COLLOQUIUM on the ferroelectric properties of single crystals, arranged by the Radio Components Research and Development Committee, was held at the Signals Research and Development Establishment, Christchurch, Hants, during September 16-17. Some sixty scientists attended in response to invitations, the Armed Services establishments, the universities and industry being represented in fairly equal numbers. The Bell Telephone Laboratories, New Jersey, which had supplied a number of large erystals for investigation by British workers, were represented by Dr. B. T. Matthias, who has achieved international reputation in this field. W. Bol and S. Duinker, representing Philips, Eindhoven, were also present.

The first paper, presented by C. F. Oxbrow (Christchureh), was prefaced with a short account of the fundamentals of the structure of barium titanate and their relation to electrical properties. Representative figures measured at Christchurch for the Bell crystals were saturation polarization, $22.5 \mu$ coulombs $/ \mathrm{cm}^{2}$; hysteresis loop area, $1.4 \times 10^{-3}$ joules $/ \mathrm{cm} .^{2}$; permittivity along $c$-axis, 300 at room temperature. The optical properties of the Bell crystals were then described. These crystals, plates $1 \times 1 \times 0.02 \mathrm{~cm}$., had a large area with the optic axis normal to the plate but were crossed by occasional laminar wedges of twinned material inclined at $45^{\circ}$ to the plate. Seen by polarized light, these wedges produced broad interference bands on a uniform background. These twin domains were removed by the application of an electric field. A colour film of this type of domain change was shown, and inferences drawn on domain magnitude and growth. The paper concluded with a description of measurements to determine the refractive index $n_{0}$ accurately, the methods described being particularly applicable to these crystals. A modification of Chaulnes's method led to a value $n_{0}=2 \cdot 49$.

P. Parsons (General Post Office Research Laboratories) exhibited photographs of the Bell crystals showing the erinkling due to the laminar domains as seen by ordinary light, the changes brought about by heating to the Curie point, and the re-establishment of the original domains by an electric field. Electrical measurements were also described. The charge/voltage hysteresis loop was particularly square; the coercive field was of the order of $1.1 \mathrm{kV} . / \mathrm{cm}$. at $50 \mathrm{c} . / \mathrm{s}$. but only $500 \mathrm{~V} . / \mathrm{cm}$. for direct current; in the region of the Curie temperature an electric field gave well-defined double or constricted loops. The behaviour of domain walls is evidently 
dependent upon the rate of change of field because measurements showed a sharp peak in the differential permittivity at a bias field equal to the coercive field. The frequency of the small measuring field was $100 \mathrm{kc} . / \mathrm{s}$. and that of the large bias field $50 \mathrm{c} . / \mathrm{s}$. These results contrast with the substantially fieldindependent value measured at Christchurch using d.c. bias.

D. Gibbs (University of Bristol) discussed domain changes. $90^{\circ}$ walls which separate regions with polar axes at $90^{\circ}$ are easily observed. $180^{\circ}$ walls separate antiparallel regions and are observable by polarized light only when additional cross-stress is applied. $180^{\circ}$ wall change, equivalent to domain reversal, takes place by nucleation and growth of spike-like domains in the direction of an applied field ${ }^{1}$. Both $90^{\circ}$ and $180^{\circ}$ wall changes are possible in single crystals. In ceramies the electrical stress set up normally precludes $180^{\circ}$ wall changes, and a parabolic $(x, D)$ relation $x=k D^{2}$ due to $90^{\circ}$ wall movements is obtained. Measurements at Bristol of electric displacement $D$ and thickness-strain $x$ produced values of $k$ up to $5 \times 10^{-5}$ e.s.u. In very pure barium titanate ceramic a flattening of the $(x, D)$ curve at the vertex could be explained by $180^{\circ}$ wall changes occurring at low field. In this pure ceramic a kink appeared in the side of the familiar $D / E$ hysteresis loop if the applied a.c. field was suddenly increased. The kink grew out at a rate dependent on temperature, and this could be evidence of the nucleation period involved in $180^{\circ}$ wall changes.

Problems which have arisen in growing single crystals of barium titanate were discussed by $\mathrm{J}$. Herbert (Plessey, Ltd.). A pure intimately mixed raw material was made by incinerating barium titanium oxalate. The Verneuil oxy-hydrogen blowtorch method was tried. Lack of success in growing large tetragonal crystals was due to the growth of crystals in the hexagonal habit. To obtain barium titanate in the tetragonal form by crystallization from a melt of titanium dioxide and barium oxide, it was necessary to use a flux. Barium chloride yielded nearly perfect crystals of barium titanate in the form of thin plates, but the yield was small. Potassium fluoride yielded crystals in the form of 'butterflies', pairs of triangular plates joined along one edge. A lively discussion followed between Dr. Matthias (Bell Laboratories), Mr. Rhodes (Standard Telecommunications Laboratories) and Mr. Herbert on the merits of the potassium fluoride method as used by J. P. Remeika ${ }^{2}$ in growing the excellent crystals described by the earlier speakers. Dr. Matthias also said that Mr. Remeika had succeeded in converting by heat treatment the hexagonal into the cubic form and vice versa. Evidently the fluoride method should be used for obtaining flat plates, while other methods such as the Verneuil might provide shapes suitable for piezoelectric investigations.

D. Campbell (Plessey, Ltd.) described major differences between crystals grown from barium chloride and potassium fluoride solvents. The potassium fluoride crystals had a surface strain layer which gave a square-net pattern under polarized light ; the pattern disappeared and then returned when the crystals were heated and cooled through the Curie temperature $\left(120^{\circ} \mathrm{C}\right.$.). The strain layer was etched away by phosphoric acid. Above the Curie temperature the optical activity of the chloride melt crystals completely disappeared, whereas some activity persisted in the case of those from fluoride melt. X-ray photographs indicated the normal con- traction of c/a ratio with temperature in agreement with observed birefringent changes for the 'chloride' crystals. The 'fluoride' crystals had very complex photographs at $85^{\circ} \mathrm{C}$. Hysteresis loops were directly dependent on complexity of structure. The American fluoride-melt crystals had ferroelectric constants agreeing with those of the chloride-melt crystals although the hysteresis loops were much more square.

In summarizing his work on sodium niobate, Dr. L. E. Cross (University of Leeds) reviewed previous work and anomalies between optical, electrical and X-ray measurements examined. His method of preparation and selection of single crystals for examination was described. Details were given of measurements of birefringence, type and arrangement of internal twins and dielectric constants over the temperature range $-195^{\circ} \mathrm{C}$. to $+650^{\circ} \mathrm{C}$. Owing to excessive conduction, observations of the effect of high electric field were given for the range $-190^{\circ} \mathrm{C}$. to $+20^{\circ} \mathrm{C}$. only. Summarizing, above $340^{\circ} \mathrm{C}$. sodium niobate was not tetragonal but had orthorhombic symmetry, and there were two further changes before it became cubic at $638^{\circ} \mathrm{C}$. The anti-ferroelectric structure at room temperature was confirmed, and there was some evidence of an additional ferroelectric polarization at low temperatures.

Two further papers on theoretical aspects of ferroelectricity were contributed by Drs. H. F. Kay and A. Devonshire (both of the University of Bristol). Dr. Kay dealt with the crystal structure of perovskite materials, with emphasis on (Cd,Ca, Sr,Ba)TiO, $(\mathrm{Li}, \mathrm{K}, \mathrm{Na}) \mathrm{NbO}_{3}$ and $(\mathrm{Li}, \mathrm{K}, \mathrm{Na}) \mathrm{TaO}_{3}$. Present knowledge is still only sufficient to indicate which materials are likely to be electrically unstable and the probable effects of substitutional replacements. The concept of ionic packing determines the probable type and magnitude of distortion at room temperature in the $\mathrm{TiO}_{6}$ octahedra characteristic of the perovskite structure. These factors can be correlated to the probable type and frequency of twinning. In barium titanate the barium ion is so large that the structure can be broken down into sheet-like Ti-O groups, accounting for the polar character. The process is essentially ionic, since recent electrical measurements of saturation polarization agree with the value obtained from X-ray data on ionic shifts. The change in character of the distortion was traced as ion size was reduced progressively from barium through strontium until finally a tetrahedron-like formation was met in non-ferroelectric cadmium titanate. The substitution of lead produced anomalies which can be partially understood. Similar arguments are in general applicable to the alkali niobates and tantalates. In sodium niobate the structure is complex, and the unit cell quadrupled. Nevertheless, its fielddependent ferroelectric behaviour can be explained.

Discussing atomic models, Dr. Devonshire said that, while physical measurements are usually conducted at constant pressure, the constants immediately derived from atomic models are those for constant volume. For the perovskites the distinction is drastic. Thus in barium titanate the ferroelectric transition at constant pressure is discontinuous, a first-order transition, while calculations indicate that at constant volume the change would probably be second order. In contrast to ferromagnetics, the dipole interaction in ferroelectrics is long-range. Various types of model were considered by Dr. Devonshire: the type appropriate to potassium dihydrogen phosphate, in which an ion can take up two or more equilibrium positions; and that appro- 
priate to barium titanate where the field in which the ion moves is anharmonic. The former gives with temperature a rapid change of $\chi$, the reciprocal susceptibility or dielectric stiffness, while the latter gives a small change. Thus, the respective values of $\partial \chi / \partial T$ are $28 \cdot 0$ and $0 \cdot 75$. The Mason model for Rochelle salt was also considered. Here two sets of ions moving in unsymmetrical fields provide, as required for this material, a polar state stable only between two temperatures. A model for anti-ferroelectricity might be built on similar lines.

Both sessions were terminated by general discussions, Dr. Matthias presiding on the first day and C. G. Garton (Electrical Research Association) on the second. The subjects at both discussions were the practical uses of barium titanate crystals, with particular emphasis on digital storage. The fundamentals of storage and methods of investigation were described. Dr. Matthias said that the Bell Laboratories are concentrating on investigations into the serious deterioration in polarization properties caused by the protracted application of unbalanced pulse trains. Mr. Oxbrow said the deterioration is amplitude-dependent and is serious only if pulse amplitudes of the order of the coercive field are used. I. Carter (Ferranti, Ltd.) reported that, after the decay, a low-frequency property, sufficient storage effect exists for high-speed operation. Also, the initial state can be restored by periodic application of a small d.c. bias. Dr. A. Robinson (Ferranti, Ltd.) compared the merits of dielectric and ferrite storage. The advantages of barium titanate storage are compactness and a more convenient impedance for use in valve circuitry. Ferrite storage has the advantages of a more advanced state of development and, although offset by elumsiness, the versatility of variable 'turns-ratio' coupling. Very recent experiments with barium titanate indicate polarization times as low as $0.5 \mu \mathrm{sec}$., so that it might permit greater speeds of operation than ferrite.

A. C. Lynch (General Post Office) said that confirmation by Merz ${ }^{1}$ that $180^{\circ}$ walls do not move laterally has removed the fear that the polarizations of adjacent crystal areas might interfere. B. de Ferranti (General Electric Co., Itd.) suggested that the non-linear characteristic of barium titanate capacitance versus field should be used in a dielectric amplifier. This would necessitate further investigation of noise in ceramics and single erystals. I. Ross (Services Electronics Research Laboratory) mentioned a possible use in infra-red switching, provided that the change in the $45^{\circ}$ laminar domains is adequately fast. Measurements have been made of the infra-red transmission of one of the Bell crystals. C. F. OxBrow

${ }^{2}$ Merz, W. J., Phys. Rev., 95, 690 (1954).

a Remeika, J. P., J. Amer. Chem. Soc., 78, 940 (1954).

\section{SHIFT WORK IN INDUSTRY}

$\mathrm{T}$

HE Institute of Persorinel Management (2-10 Hill Strect, London, W.1) has issued a broadsheet surveying the issues which affect the application of shift systems in industry. The broadsheet has been prepared by F. P. Cook, of Courtaulds, Ltd., but is based upon information obtained from a large number of different industries.

Among the conclusions brought out in the survey is that the economic advantages of shift work are greatest where capital charges and overheads are high in relation to labour costs. The greater the proportion of the total cost of production that is occupied by labour costs, the less the economic advantages of shift work become. When shift work is first introduced in a discontinuous process where employment has previously been on day work, the double-day shift system is probably the most advantageous for all concerned-for management because it is a second shift which offers the greatest alleviation to fixed charges, and for employees because the physical and social disadvantages are much less than those of shift systems which involve night work. The appeal of shift work to employees depends largely on how attractive it is made financially. Although it is only one factor, shift work can prove a sound economic way of meeting the natural urge towards shorter hours of work and higher earnings.

Quite apart from the ballot which is usually required by law before women and young persons can be employed on double day-shift work, the investigations show that full explanation and consultation are needed before any successful shift system can be introduced. Of all shift systems, those involving night work are most likely to have a deleterious effect on health; this is especially applicable to systems which involve permanent night work. Any unfortunate effects will vary with individuals, the nature and hours of their work, and their habits outside the factory, so that it would be hard to establish a general rule, even if more evidence were available. For most employees, shift work involves a balancing of material gain against social loss ; thus, in pursuing the possible economic advantages of the extension of shift work, which can only be a gradual process, those in authority must take the possible social drawbacks into account.

Mr. Cook concludes that one of the temptations of these complicated times is to over-simplify, and this is typified by the search for the single solution to any problem. In personnel management, recent years have seen the fashionable development of joint consultation, job evaluation, communications, financial incentives, financial enlightenment, merit rating of management development, and work study. Despite the creation of much pseudo-technical supporting jargon, not one of these is a cure-all by itself, and together they can be no more than further aids to the vastly complicated business of modern industrial management. The extension of shift work will not alone revive the fortunes of a nation or concern; but, in the right circumstances, it can help.

\section{FORESTRY COMMISSION REPORT FOR THE YEAR 1952-53}

IN the operations of the Forestry Commission during the year $1952-53^{*}$, economy in spending was a guiding factor in deciding the programme of work. In order to carry out the expanding programme of planting and thinnings in the young plantations with the funds available, it was necessary to postpone work such as building new houses and new roads. Many of the forest services of the British.

* Forestry Commission. Thirty-fourth Annual Report of the Forestry Commissioners for the Year ended September 30th, 1953. Pp. 84. (London: H.M.S.O, 1954.) 3s. net. 